\title{
GENERALIZED SPHERICAL HARMONICS
}

BY

\author{
M. H. PROTTER(1)
}

1. Introduction. In two recent papers Bers and Gelbart $\left([3]\right.$ and $\left.[4]\left({ }^{2}\right)\right)$ considered certain classes of functions which are solutions of the differential equation

$$
\left(\frac{\sigma_{1}(x)}{\tau_{1}(y)} u_{x}\right)_{x}+\left(\frac{\sigma_{2}(x)}{\tau_{2}(y)} u_{y}\right)_{y}=0 .
$$

Their approach to these classes of functions was based on the Cauchy-Riemann equations and the theory of functions of a complex variable. Certain functions, called sigma-monogenic functions, were developed which satisfied generalized Cauchy-Riemann equations; the real parts of these functions satisfied equation (1.1). The sigma-monogenic functions were shown to possess many of the properties of analytic functions of a complex variable and, in particular, to have power series developments in terms of generalized powers $a \cdot Z^{(n)}$.

Any direct attempt at extending these results to second order equations in three variables must fail because of the lack of a corresponding theory of a complex variable. However, the generalized powers $X^{(n)}$ and $Y^{(n)}$, from which the generalized powers $Z^{(n)}$ were formed, can be arrived at without touching the study of complex variables. In equation (1.1) suppose a solution $u$ exists of the form $u(x, y)=X(x) Y(y)$. Let the initial conditions $X(0)=Y(0)$ $=1, X^{\prime}(0)=Y^{\prime}(0)=0$ be imposed. The substitution of $u$ into equation (1.1) yields two ordinary differential equations which may be transformed into integral equations. If the initial conditions are taken into account and a process of iteration is applied to the integral equations, the powers $X^{(n)}$ and $Y^{(n)}$ are obtained as coefficients of certain expansions; these are precisely the powers obtained by Bers and Gelbart. Their results can be extended by the method of approach described above.

The present paper considers classes of solutions of equations $\left(^{3}\right)$ of the form

Presented to the Society, August 23, 1946; received by the editors July 29, 1946.

(1) The author wishes to express his appreciation to Professor Lipman Bers for his considerable help and constant encouragement in the development of this paper.

(2) Numbers in brackets refer to the bibliography at the end of the paper.

(3) By a different process Bergman $[1 ; 7]$ introduced methods for generating and investigating functions satisfying equation (1.1). His methods can also be generalized to yield results in three variables. However, he only indicated in an abstract [2] how particular results can be obtained for equations which represent a special case of the equations considered in this paper. 


$$
\left\{\sigma_{1}(x) \tau_{1}(y) \pi_{1}(z) u_{x}\right\}_{x}+\left\{\sigma_{2}(x) \tau_{2}(y) \pi_{2}(z) u_{y}\right\}_{y}+\left\{\sigma_{3}(x) \tau_{3}(y) \pi_{3}(z) u_{z}\right\}_{z}=0 .
$$

Certain generalized powers $X^{(k)}, Y^{(l)}, Z^{(m, n)}$ are obtained and a class of functions, $\bar{R}_{n, \nu}(X, Y, Z)$, "polynomials" in these generalized powers, is formed. It is shown that the functions $\bar{R}_{n, \nu}(X, Y, Z)$ possess the following properties.

(1) For each $n$ and $\nu, \bar{R}_{n, \nu}$ is a solution of equation (1.2) which is regular in the entire space.

(2) The functions $\bar{R}_{n, \nu}$ can be computed by quadratures.

(3) Every solution $u(x, y, z)$ of equation (1.2) regular in the neighborhood of the origin can be expanded uniquely in an absolutely convergent series of the form

$$
u(x, y, z)=\sum_{n=0}^{\infty} \sum_{\nu=0}^{2 n} \frac{\alpha_{n, \nu}}{n !} \bar{R}_{n, \nu}(X, Y, Z)
$$

where the coefficients $\alpha_{n, v}$ are given by certain simple formulas.

(4) Every solution $u(x, y, z)$ of equation (1.2) regular in the neighborhood of any point $P(x, y, z)$ can be expanded in a series of the same powers $\bar{R}_{n, \nu}(X, Y, Z)$ of the form

$$
u(x, y, z)=\sum_{n=0}^{\infty} \sum_{\nu=0}^{2 n} \frac{\alpha_{n, \nu}}{n !} \sum_{m=0}^{n} \sum_{\mu=0}^{2 m} \frac{\beta_{m, \mu}}{m !} \bar{R}_{m, \mu}(X, Y, Z),
$$

where the coefficients $\alpha_{n, \nu}$ and $\beta_{m, \mu}$ are given by formulas similar to the $\alpha_{n, \nu}$ of (3) above, the $\beta_{m, \mu}$ being independent of $u(x, y, z)$.

In the special case in which equation (1.2) reduces to the Laplace equation in rectangular coordinates, the functions $\bar{R}_{n, \nu}$ become homogeneous harmonic polynomials in $x, y$, and $z$ of degree $n$. Any harmonic function regular in the neighborhood of a point can be expanded in an absolutely convergent series of the form

$$
\sum_{n=0}^{\infty} \sum_{\nu=0}^{2 n} \frac{\alpha_{n, \nu}}{n !} \bar{R}_{n, \nu}(x, y, z)
$$

where the coefficients $\alpha_{n, \nu}$ are given by Taylor formulas.

2. On a class of spherical harmonics. The Laplace equation

$$
u_{x x}+u_{y y}+u_{z z}=0
$$

has the solution $u(x, y, z)=e^{\alpha x} e^{\beta y} e^{i \gamma z}$ if the relation

$$
\alpha^{2}+\beta^{2}-\gamma^{2}=0
$$

holds. The function $u$ may be considered as an analytic function of $\alpha, \beta$, and $\gamma$, and as such it may be expanded in a power series in these variables. From (2.2), the relations $\gamma^{2 m}=\left(\alpha^{2}+\beta^{2}\right)^{m}$ and $\gamma^{2 m+1}=\gamma\left(\alpha^{2}+\beta^{2}\right)^{m}$ hold. Hence, the expansion for $u$ may be written in the form 


$$
u=\sum_{n=0}^{\infty} \sum_{\nu=0}^{n} \frac{\alpha^{\nu} \beta^{n-\nu}}{n !}\left\{R_{n, 2 \nu}(x, y, z)+i \frac{\gamma}{n+1} R_{n+1,2 v+1}(x, y, z)\right\}
$$

where the coefficients $R_{n, \nu}$ are functions of $x, y$, and $z$. In fact it is easily seen that they are polynomials. Since $u$, considered as a function of $x, y$, and $z$, is a solution of the Laplace equation for all values of $\alpha$ and $\beta$ if $\gamma^{2}=\alpha^{2}+\beta^{2}$, each coefficient in the expansion (2.3) must be a harmonic function. Hence $R_{n, v}$ is a harmonic polynomial for each $n$ and $\nu$.

Explicit expressions for the polynomials $R_{n, v}(x, y, z)$ may be derived in the following manner. First the function $u(x, y, z)=e^{\alpha x} e^{\beta y} e_{2}{ }^{i \gamma z}$ may be expanded in the form

$$
u(x, y, z)=\sum_{k=0}^{\infty} \frac{\alpha^{k} x^{k}}{k !} \sum_{l=0}^{\infty} \frac{\beta^{l} y^{l}}{l !} \sum_{m=0}^{\infty} \frac{(i \gamma)^{m_{z}} z^{m}}{m !} .
$$

Then a rearrangement yields

$$
u(x, y, z)=\sum_{k, l=0}^{\infty} \frac{\alpha^{k} \beta^{l} x^{k} y^{l}}{k ! l !} \sum_{m=0}^{\infty}(-1)^{m} \gamma^{2 m}\left\{\frac{z^{2 m}}{(2 m) !}+i \gamma \frac{z^{2 m+1}}{(2 m+1) !}\right\} .
$$

From the binomial theorem we have

$$
\gamma^{2 m}=\left(\alpha^{2}+\beta^{2}\right)^{m}=\sum_{p=0}^{m} \frac{m !}{p !(m-p) !} \alpha^{2 m-2 p} \beta^{2 p},
$$

and the expansion for $u(x, y, z)$ becomes

$$
u=\sum_{k, l, m=0}^{\infty} \sum_{p=0}^{m} \frac{(-1)^{m} m ! \alpha^{k+2 m-2 p} \beta^{l+2 p} x^{k} y^{l}}{k ! l ! p !(m-p) !}\left\{\frac{z^{2 m}}{(2 m) !}+i \gamma \frac{z^{2 m+1}}{(2 m+1) !}\right\} .
$$

Consider first the terms of (2.4) for which $k$ and $l$ are both even. Let $k=2 r, l=2 s, N=r+m+s, n=2 N$, and $\nu=r+m-p$. Hence the terms of (2.4) for which both $k$ and $l$ are even can be written

$$
\begin{aligned}
\sum_{N=0}^{\infty} \sum_{\nu=0}^{N} \frac{\alpha^{2 \nu} \beta^{2 N-2 \nu}}{(2 N) !} \sum_{s=0}^{N-\nu} \sum_{r=0}^{\nu} \frac{(-1)^{N-r-s}(2 N) !(N-r-s) ! x^{2 r} y^{2 s}}{(2 r) !(2 s) !(N-\nu-s) !(\nu-r) !} \\
\cdot\left\{\frac{z^{2 N-2 r-2 s}}{(2 N-2 r-2 s) !}+i \gamma \frac{z^{2 N-2 r-2 s+1}}{(2 N-2 r-2 s+1) !}\right\} .
\end{aligned}
$$

Since the above expression is in the form of (2.3), the explicit expressions for $R_{n, \nu}(x, y, z)$ for the case $k$ and $l$ even are

$$
\begin{aligned}
& R_{2 N, 4 \nu}(x, y, z) \\
& =\sum_{s=0}^{N-r} \sum_{r=0}^{\nu} \frac{(-1)^{N-r-s}(2 N) !(N-r-s) ! x^{2 r} y^{2 s} z^{2 N-2 r-2 s}}{(2 r) !(2 s) !(2 N-2 r-2 s) !(N-\nu-s) !(\nu-r) !}
\end{aligned}
$$


and

$$
\begin{aligned}
& R_{2 N+1,4 v+1}(x, y, z) \\
& =\sum_{s=0}^{N \rightarrow n} \sum_{r=0}^{n} \frac{(-1)^{N-r-s}(2 N+1) !(N-r-s) ! x^{2 r} y^{2 s} z^{2 N-2 r-2 s+1}}{(2 r) !(2 s) !(2 N-2 r-2 s+1) !(N-\nu-s) !(\nu-r) !}
\end{aligned}
$$

in which $\nu=0,1,2, \cdots, N$ and $N=0,1,2, \cdots$.

For the remaining combinations of $k$ and $l$ even and odd the expressions for $R_{n, v}(x, y, z)$ are

$$
\begin{aligned}
& R_{2 N, 4 v+2}(x, y, z) \\
& =\sum_{r=1}^{N \rightarrow n} \sum_{r=0}^{n} \frac{(-1)^{N-r-s}(2 N) !(N-r-s) ! x^{2 r+1} y^{2 s-1} z^{2 N-2 r-2 s}}{(2 r+1) !(2 s-1) !(N-\nu-s) !(\nu-r) !(2 N-2 r-2 s) !},
\end{aligned}
$$

$R_{2 N+1,4 v+3}(x, y, z)$

$$
=\sum_{s=1}^{N-p} \sum_{r=0}^{n} \frac{(-1)^{N-r-s}(2 N+1) !(N-r-s) ! x^{2 r+1} y^{2 s-1} z^{2 N-2 r-2 s+1}}{(2 r+1) !(2 s-1) !(2 N-2 r-2 s+1) !(N-\nu-s) !(\nu-r) !},
$$

$R_{2 N+1,4 v}(x, y, z)$

$$
=\sum_{s=0}^{N-p} \sum_{r=0}^{n} \frac{(-1)^{N-r-s}(2 N+1) !(N-r-s) ! x^{2 r} y^{2 s+1} z^{2 N-2 r-2 s}}{(2 r) !(2 s+1) !(2 N-2 r-2 s) !(N-\nu-s) !(\nu-r) !},
$$

$R_{2 N+2,4 v+1}(x, y, z)$

$$
=\sum_{s=0}^{N-p} \sum_{r=0}^{p} \frac{(-1)^{N-r-s}(2 N+2) !(N-r-s) ! x^{2 r} y^{2 s+1} z^{2 N-2 r-2 s+1}}{(2 r) !(2 s+1) !(2 N-2 r-2 s+1) !(N-\nu-s) !(\nu-r) !},
$$

$R_{2 N+1,4 v+2}(x, y, z)$

$$
=\sum_{s=0}^{N-p} \sum_{r=0}^{n} \frac{(-1)^{N-r-s}(2 N+1) !(N-r-s) ! x^{2 r+1} y^{2 s} z^{2 N-2 r-2 s}}{(2 r+1) !(2 s) !(2 N-2 r-2 s) !(N-\nu-s) !(\nu-r) !},
$$

$R_{2 N+2,4 v+3}(x, y, z)$

$$
=\sum_{s=0}^{N-p} \sum_{r=0}^{v} \frac{(-1)^{N-r-s}(2 N+2) !(N-r-s) ! x^{2 r+1} y^{2 s} z^{2 N-2 r-2 o+1}}{(2 r+1) !(2 s) !(N-\nu-s) !(\nu-r) !(2 N-2 r-2 s+1) !} .
$$

(2.6) THeorem. For each $n$, the quantities $R_{n, v}(x, y, z)$ form a set of $2 n+1$ homogeneous, linearly independent, harmonic polynomials of degree $n$.

Proof. It has already been shown that each $R_{n, v}(x, y, z)$ is a solution of the Laplace equation. The expressions (2.5) show that $R_{n, v}(x, y, z)$ is a homogeneous polynomial in $x, y$, and $z$ of degree $n$ for each value of $\nu$ and all $n$.

An enumeration shows that, for each $n$, there are $2 n+1$ polynomials of degree $n$. For $n$ even $(=2 N)$ there are $N+1$ polynomials obtained from (2.5a), $N$ polynomials from (2.5c), $N$ from (2.5f), and $N$ from (2.5h). Hence there 
are $2 n+1$ polynomials in all. Similarly, an examination of expressions (2.5) yields the result for $n$ odd.

The linear independence remains to be shown. Consider (2.5a) for a fixed $N$ and $\nu$. The expression for $R_{2 N, 4 \nu}(x, y, z)$ contains as one term the monomial

$$
\frac{(2 N) ! x^{2 \nu} y^{2 N-2 \nu}}{(2 \nu) !(2 N-2 \nu) !}
$$

which is obtained by setting $r=\nu$ and $s=N-\nu$. All other terms in the expression for $R_{2 N, 4 \nu}(x, y, z)$ contain $z$ to some positive power. Now consider $R_{2 N, 4 \nu^{\prime}}(x, y, z)$ where $\nu^{\prime} \neq \nu$. The monomial not containing $z$ to some positive power is

$$
\frac{(2 N) ! x^{2 \nu^{\prime}} y^{2 N-2 \nu^{\prime}}}{\left(2 \nu^{\prime}\right) !\left(2 N-2 \nu^{\prime}\right) !}
$$

If $a_{1}$ and $a_{2}$ are constants, then $a_{1} R_{2 N, 4 \nu}+a_{2} R_{2 N, 4 \nu^{\prime}}=0$ only if $a_{1}$ and $a_{2}$ are both zero. This follows from the fact that two of the terms are expressions (2.7) and (2.8) and all the remaining terms contain $z$ to a positive power. Thus the polynomials $R_{2 N, 4 \nu}(x, y, z), \nu=0,1,2, \cdots, N$, are linearly independent. In a similar way each of the sets $(2.5 \mathrm{~b})$ to $(2.5 \mathrm{~h})$ forms a linearly independent set. The expressions (2.5) for the polynomials $R_{n, \nu}(x, y, z)$ show at once the following oddness and evenness relations:

$$
\begin{aligned}
& R_{n, \nu}(x, y, z)=(-1)^{[\nu / 2]} R_{n, \nu}(-x, y, z), \\
& R_{n, \nu}(x, y, z)=(-1)^{[n+\nu / 2+1 / 2]} R_{n, \nu}(x,-y, z), \\
& R_{n, \nu}(x, y, z)=(-1)^{\nu} R_{n, \nu}(x, y,-z) .
\end{aligned}
$$

Therefore no set can contain a polynomial which is a linear combination of polynomials from other sets. Thus the $2 n+1$ polynomials of degree $n$ are linearly independent.

(2.10) Lemma. The polynomials $R_{n, v}(x, y, z)$ satisfy the differential recursion formulas

$$
\begin{gathered}
\frac{\partial}{\partial x} R_{n, \nu}=n R_{n-1, v-2}, \\
\frac{\partial}{\partial y} R_{n, \nu}=n R_{n-1, \nu}, \\
\frac{\partial}{\partial z} R_{n, 2 v+1}=n R_{n-1,2 v,} \frac{\partial}{\partial z} R_{n, 2 v}=-n\left(R_{n-1,2 v-3}+R_{n-1,2 v+1}\right) .
\end{gathered}
$$

Proof. From the defining relation $u(x, y, z)=e^{\alpha x} e^{\beta y} e^{i \gamma z}$, it follows that $u_{x}=\alpha u, u_{y}=\beta u, u_{z}=i \gamma u$. The relation $u_{x}=\alpha u$ implies that 


$$
\begin{aligned}
\sum_{n=0}^{\infty} \sum_{\nu=0}^{n} \frac{\alpha^{\nu} \beta^{n-\nu}}{n !} \frac{\partial}{\partial x}\left\{R_{n, 2 \nu}+\frac{i \gamma}{n+1}\right. & \left.R_{n+1,2 \nu+1}\right\} \\
& =\sum_{n=0}^{\infty} \sum_{\nu=0}^{n} \frac{\alpha^{\nu+1} \beta^{n-\nu}}{n !}\left\{R_{n, 2 \nu}+\frac{i \gamma}{n+1} R_{n+1,2 \nu+1}\right\} .
\end{aligned}
$$

This equation is an identity in $\alpha, \beta$, and $\gamma$; hence, coefficients of corresponding terms must be equal. Therefore we have

$$
\frac{\partial}{\partial x} R_{n, 2 \nu}=\frac{n !}{(n-1) !} R_{n-1,2 v-2}, \frac{\partial}{\partial x} R_{n+1,2 v+1}=\frac{(n+1) !}{n !} R_{n, 2 \nu-1} .
$$

Formula (2.11a) follows at once from these relations. In a similar way $(2.11 \mathrm{~b})$ and $(2.11 \mathrm{c})$ are obtained.

If we apply the recursion formulas twice in (2.11a), (2.11b), and (2.11c), and add, we get $\Delta R_{n, v}(x, y, z)=0$, a fact already obtained.

(2.12) Lemma. For all $n$ and $\nu$ the inequality $\left({ }^{4}\right)$

$$
\left|R_{n, v}(x, y, z)\right| \leqq 2^{n}(|x|+|y|+|z|)^{n}
$$

holds.

Proof. Consider, for example, $R_{2 N, 4 v}(x, y, z)$. From (2.5a) we have

$$
{ }_{\mid} R_{2 N, 4 \nu}(x, y, z) \mid \leqq \sum_{s=0}^{N-\nu} \sum_{r=0}^{\nu} \frac{(2 N) !|x|^{2 r}|y|^{2 s}|z|^{2 N-2 r-2 s}(N-r-s) !}{(2 r) !(2 s) !(2 N-2 r-2 s) !(N-\nu-s) !(\nu-r) !} .
$$

From the inequality

$$
\frac{(N-r-s) !}{(N-\nu-s) !(\nu-r) !} \leqq \sum_{\nu=0}^{N-r-s} \frac{(N-r-s) !}{(N-\nu-s) !(\nu-r) !}=2^{N-r-s} \leqq 2^{N}
$$

it follows that

$$
\left|R_{2 N, 4 v}(x, y, z)\right| \leqq 2^{N} \sum_{s=0}^{N-v} \sum_{r=0}^{\nu} \frac{(2 N) !|x|^{2 r}|y|^{2 s}|z|^{2 N-2 r-2 s}}{(2 r) !(2 s) !(2 N-2 r-2 s) !} .
$$

On the other hand, we have

$$
\begin{aligned}
(|x|+|y|+|z|)^{2 N} & =\sum_{m=0}^{2 N} \sum_{p=0}^{2 N-m} \frac{(2 N) !|x|^{m}|y|^{p}|z|^{2 N-m-p}}{m ! p !(2 N-m-p) !} \\
& \geqq \sum_{s=0}^{N-\nu} \sum_{r=0}^{\nu} \frac{(2 N) !|x|^{2 r}|y|^{2 s}|z|^{2 N-2 r-2 s}}{(2 r) !(2 s) !(2 N-2 r-2 s) !} .
\end{aligned}
$$

Hence we obtain

(4) This inequality is crude but sufficient for our purposes. 


$$
\left|R_{2 N, 4 \nu}(x, y, z)\right| \leqq 2^{N}(|x|+|y|+|z|)^{2 N} \leqq 2^{2 N}(|x|+|y|+|z|)^{2 N} .
$$

In a similar way it is easily shown that each of the other formulas (2.5b)$(2.5 \mathrm{~h})$ satisfies the same inequality.

Suppose $u(x, y, z)$ is a harmonic function, regular in the neighborhood of the origin. Let us define the auxiliary functions

$$
\begin{gathered}
u_{1}(x, y)=u(x, y, 0), \quad u_{2}(x, y)=\left.\frac{\partial}{\partial z} u(x, y, z)\right|_{z=0}, \\
u_{3}(x, y)=u_{1}(x, y)+u_{2}(x, y) .
\end{gathered}
$$

We consider a series of the form

$$
\sum_{n=0}^{\infty} \sum_{\nu=0}^{2 n} \frac{\alpha_{n, \nu}}{n !} R_{n, \nu}(x, y, z)
$$

in which the coefficients are given by the relations

$$
\begin{aligned}
& \alpha_{n, 2 \nu}=\left.\frac{\nu !(n-\nu) !}{n !} \frac{\partial^{n} u_{1}(x, y)}{\partial x^{\nu} \partial y^{n-\nu}}\right|_{x=y=0}, \\
& \nu=0,1,2, \cdots, n ; n=0,1,2, \cdots . \\
& \alpha_{n, 2 v+1}=\left.\frac{\nu !(n-\nu-1) !}{(n-1) !} \frac{\partial^{n-1} u_{2}(x, y)}{\partial x^{\nu} \partial y^{n-\nu-1}}\right|_{x=y=0}, \\
& \nu=0,1,2, \cdots, n-1 ; n=0,1,2, \cdots .
\end{aligned}
$$

(2.15) Theorem. Let $u(x, y, z)$ be regular in the neighborhood of the origin and satisfy the equation $\Delta u=0$. Then $u(x, y, z)$ can be expanded in a series

$$
u(x, y, z)=\sum_{n=0}^{\infty} \sum_{\nu=0}^{2 n} \frac{\alpha_{n, v}}{n !} R_{n, v}(x, y, z)
$$

in which the coefficients are given by (2.14). Further, the series

$$
\sum_{n=0}^{\infty} \sum_{\nu=0}^{2 n}\left|\frac{\alpha_{n, \nu}}{n !} R_{n, \nu}(x, y, z)\right|
$$

converges uniformly in a neighborhood of the origin.

Proof. Since $u(x, y, z)$ is regular in the neighborhood of the origin it can be expanded in a Taylor series which converges absolutely and uniformly in the neighborhood of the origin. Hence, the terms of this Taylor series can be rearranged, and we have

$$
u(x, y, z)=\sum_{n=0}^{\infty}\left\{\sum_{\nu=0}^{2 n} \frac{\alpha_{n, \nu}}{n !} R_{n, \nu}(x, y, z)\right\} .
$$


The relations (2.14) are obtained by consideration of the polynomials $\left.R_{n, v}(x, y, z)\right|_{z=0}$ and $\left.(\partial / \partial z) R_{n, y}(x, y, z)\right|_{z=0}$. An examination of the expressions (2.5a) $-(2.5 \mathrm{~h})$ shows that

$$
\begin{gathered}
R_{n, 2 v+1}(x, y, 0)=0, \quad R_{n, 2 v}(x, y, 0)=\frac{n ! x^{\nu} y^{n-\nu}}{\nu !(n-\nu) !} \\
\left.\frac{\partial}{\partial z} R_{n, 2 \nu}(x, y, z)\right|_{z=0}=0,\left.\quad \frac{\partial}{\partial z} R_{n, 2 v+1}(x, y, z)\right|_{z=0}=\frac{n ! x^{\nu} y^{n-\nu-1}}{\nu !(n-\nu-1) !}
\end{gathered}
$$

for all $n$ and $\nu$. The function $u_{8}(x, y)$ thus has the expansion

$$
\text { (2.17) } u_{3}(x, y)=\sum_{n=0}^{\infty} \sum_{\nu=0}^{n} \alpha_{n, 2 v} \frac{x^{\nu} y^{n-\nu}}{\nu !(n-\nu) !}+\sum_{n=0}^{\infty} \sum_{\nu=0}^{n-1} \alpha_{n, 2 v+1} \frac{x^{\nu} y^{n-\nu-1}}{\nu !(n-\nu-1) !} \cdot
$$

On the other hand $u_{3}(x, y)$, as an analytic function of $x$ and $y$ in the neighborhood of the origin, has the Taylor expansion

$$
u_{3}(x, y)=\sum_{n=0}^{\infty} \sum_{p=0}^{n} \frac{A_{n, v} x^{p} y^{n-v}}{n !}
$$

in which

$$
A_{n, \nu}=\left.\frac{\partial^{n} u_{3}(x, y)}{\partial x^{\nu} \partial y^{n-\nu}}\right|_{x=y=0} .
$$

The formulas (2.14) for $\alpha_{n, v}$ are obtained by equating coefficients in like terms of (2.17) and (2.18). Now suppose the series (2.13) converges for $|x|+|y|$ $+|z| \leqq r_{0}$. Then from Lemma (2.12) we have

$$
\left|R_{n, y}(x, y, z)\right| \leqq\left(2 r_{0}\right)^{n}
$$

for all $n$ and $\nu$. Hence if (2.14) is taken into account it follows that

$$
\begin{aligned}
\sum_{n=0}^{\infty} \sum_{\nu=0}^{2 n}\left|\frac{\alpha_{n, \nu}}{n !}\right| \cdot\left|R_{n, \nu}(x, y, z)\right| & \leqq \sum_{n=0}^{\infty} \sum_{\nu=0}^{2 n}\left|\frac{\alpha_{n, \nu}}{n !}\right|\left(2 r_{0}\right)^{n} \\
& =\sum_{n=0}^{\infty} \sum_{\nu=0}^{2 n}\left|\frac{\nu !(n-\nu) !}{n !} \cdot \frac{A_{n, \nu}}{n !}\right| \cdot\left(2 r_{0}\right)^{n} \\
& \leqq \sum_{n=0}^{\infty} \sum_{\nu=0}^{2 n}\left|\frac{A_{n, \nu}}{n !}\right|\left(2 r_{0}\right)^{n} .
\end{aligned}
$$

But since the Taylor series for $u_{3}(x, y)$ converges absolutely and uniformly, we have for sufficiently small $r$

$$
\sum_{n=0}^{\infty} \sum_{\nu=0}^{2 n}\left|\frac{\alpha_{n, \nu}}{n !}\right| \cdot\left|R_{n, \nu}(x, y, z)\right| \leqq \sum_{n=0}^{\infty} \sum_{\nu=0^{-}}^{2 n}-A_{n, \nu} \mid\left(2 r_{0}\right)^{n}<M .
$$


3. Condition for solvability by separation of variables. Bers and Gelbart, in their papers [3] and [4], considered a class of functions defined by solutions of the equation

$$
\left\{\frac{\sigma_{1}(x)}{\tau_{1}(y)} u_{x}\right\}_{x}+\left\{\frac{\sigma_{2}(x)}{\tau_{2}(y)} u_{y}\right\}_{y}=0 .
$$

This second order equation in two variables is characterized by the matrix

$$
\Sigma_{1}=\left\|\begin{array}{ll}
\sigma_{1}(x) & \tau_{1}(y) \\
\sigma_{2}(x) & \tau_{2}(y)
\end{array}\right\|
$$

In this section we shall be concerned with solutions of the second order equation in three variables

$$
\left\{\sigma_{1}(x) \tau_{1}(y) \pi_{1}(z) u_{x}\right\}_{x}+\left\{\sigma_{2}(x) \tau_{2}(y) \pi_{2}(z) u_{y}\right\}_{y}
$$

$$
+\left\{\sigma_{3}(x) \tau_{3}(y) \pi_{3}(z) u_{z}\right\}_{z}=0,
$$

characterized by the matrix

$$
\Sigma_{2}=\left\|\begin{array}{ccc}
\sigma_{1}(x) & \tau_{1}(y) & \pi_{1}(z) \\
\sigma_{2}(x) & \tau_{2}(y) & \pi_{2}(z) \\
\sigma_{3}(x) & \tau_{3}(y) & \pi_{3}(z)
\end{array}\right\|
$$

If the functions $\sigma_{i}(x), \tau_{i}(y), \pi_{i}(z), i=1,2,3$, are arbitrary functions of the variables $x, y, z$ respectively (with sufficient differentiability properties), then (3.1) will not, in general, admit solutions $u$ of the form $u=\Phi(x) \Psi(y) \mathrm{X}(z)$. This is shown in the following theorem.

(3.3) THEOREM. Let the coefficients $\sigma_{i}(x), \tau_{i}(y), \pi_{i}(z), i=1,2,3$, of equation (3.1) be analytic $\left(^{5}\right)$ functions of their respective variables. Then a necessary and sufficient condition that (3.1) admit nontrivial( $\left.{ }^{6}\right)$ solutions of the form $u=\Phi(x) \Psi(y) \mathbf{X}(z)$ is that at least two of the three conditions

$$
\frac{\sigma_{3}(x)}{\sigma_{2}(x)}=\text { const., } \quad \frac{\tau_{1}(y)}{\tau_{3}(y)}=\text { const., } \quad \frac{\pi_{1}(z)}{\pi_{2}(z)}=\text { const. }
$$

hold.

Proof. The sufficiency will be shown in $\$ 4$ by the simple method of exhibiting a class of solutions of equation (3.1) in which conditions (3.4) hold.

To prove the necessity suppose (3.1) admits a solution of the form

(5) It is sufficient to assume, for example, that the functions $\sigma_{i}(x), \tau_{i}(y), \pi_{i}(z), i=1,2,3$, have continuous first derivatives. However the applications of this theorem will be to cases in which the functions are already analytic.

$\left.{ }^{(}\right)$A trivial solution would be one in which one or more of the functions $\Phi, \Psi, X$ are constant or linear in their respective variables. 
$u=\Phi(x) \Psi(y) \mathrm{X}(z)$. This solution, when substituted in (3.1), yields

$$
\begin{aligned}
\sigma_{1}(x) \tau_{1}(y) \pi_{1}(z) \frac{\Phi^{\prime \prime}(x)}{\Phi(x)} & +\sigma_{1}^{\prime}(x) \tau_{1}(y) \pi_{1}(z) \frac{\Phi^{\prime}}{\Phi}+\sigma_{2}(x) \tau_{2}(y) \pi_{2}(z) \frac{\Psi^{\prime \prime}(y)}{\Psi(y)} \\
& +\sigma_{2}(x) \tau_{2}^{\prime}(y) \pi_{2}(z) \frac{\Psi^{\prime \prime}}{\Psi}+\sigma_{3}(x) \tau_{3}(y) \pi_{3}(z) \frac{X^{\prime \prime}(z)}{X(z)} \\
& +\sigma_{3}(x) \tau_{3}(y) \pi_{3}^{\prime}(z) \frac{X^{\prime}}{X}=0 .
\end{aligned}
$$

If this last equation is divided by $\sigma_{3}(x) \tau_{3}(y) \pi_{1}(z)$ and then differentiated with respect to $z$, the equation

$$
\frac{\sigma_{2}(x)}{\sigma_{3}(x)}\left\{\frac{\tau_{2}(y) \Psi^{\prime \prime}}{\tau_{3}(y) \Psi}+\frac{\tau_{2}^{\prime}(y)}{\tau_{3}(y)}\right\} \frac{d}{d z}\left(\frac{\pi_{2}(z)}{\pi_{1}(z)}\right)+\frac{d}{d z}\left\{\frac{\pi_{3}(z) \mathrm{X}^{\prime \prime}}{\pi_{1}(z) \mathrm{X}}+\frac{\pi_{3}^{\prime}(z) \mathrm{X}^{\prime}}{\pi_{1}(z) \mathrm{X}}\right\}=0
$$

is obtained. Equation (3.6) states that the product of a function of $x$, a function of $y$, and a function of $z$ is equal to a function of $z$. This can hold only if one of the following two cases prevails.

Case 1. $\pi_{2}(z) / \pi_{1}(z)=$ const.

Case 2.

$$
\frac{\sigma_{2}(x)}{\sigma_{3}(x)}\left\{\frac{\tau_{2}(y) \Psi^{\prime \prime}}{\tau_{3}(y) \Psi}+\frac{\tau_{2}^{\prime}(y) \Psi^{\prime}}{\tau_{3}(y) \Psi}\right\}=\text { const. }
$$

First let us suppose that $\pi_{2}(z) / \pi_{1}(z)=$ constant. Without loss of generality we may let this constant be unity. Then, from (3.6), we find

$$
\frac{\pi_{3}(z) \mathrm{X}^{\prime \prime}}{\pi_{1}(z) \mathrm{X}}+\frac{\pi_{3}^{\prime}(z) \mathrm{X}^{\prime}}{\pi_{1}(z) \mathrm{X}}=\text { const. }=c_{1},
$$

and

$$
\frac{d}{d z}\left\{\pi_{3}(z) \mathrm{X}^{\prime}(z)\right\}=c_{1} \pi_{1}(z) \mathrm{X}(z)
$$

Hence, we have

$$
\pi_{3}(z) \mathrm{X}^{\prime}(z)=c_{1} \int^{z} \pi_{1}(z) \mathrm{X}(z) d z+c_{2}
$$

and

$$
\mathrm{X}(z)=c_{1} \int^{z} \frac{1}{\pi_{3}(\xi)} \int^{\xi} \pi_{1}(\eta) \mathrm{X}(\eta) d \eta d \xi+c_{2} \int^{z} \frac{d \xi}{\pi_{3}(\xi)}+c_{3}
$$

If expression (3.5) is divided by $\sigma_{2}(x) \tau_{2}(y) \pi_{1}(z)$ and then differentiated with 
respect to $x$, and if (3.7) is taken into account, the equation

$$
\frac{d}{d x}\left\{\frac{\sigma_{1}(x) \Phi^{\prime \prime}}{\sigma_{2}(x) \Phi}+\frac{\sigma_{1}^{\prime}(x) \Phi^{\prime}}{\sigma_{2}(x) \Phi}\right\}+\frac{\tau_{3}(y)}{\tau_{1}(y)} \frac{d}{d x}\left\{\frac{\sigma_{3}(x)}{\sigma_{2}(x)}\right\}=0
$$

results. Equation (3.9) implies that either $\tau_{3}(y) / \tau_{1}(y)=$ const. or $\sigma_{3}(x) / \sigma_{2}(x)$ $=$ const. In either case the result follows. For the case $\sigma_{3}(x)=\sigma_{2}(x)$, the explicit expression for $\Phi(x)$ is

$$
\Phi(x)=k_{1} \int^{x} \frac{1}{\sigma_{1}(\xi)} \int^{\xi} \sigma_{2}(\eta) \Phi(\eta) d \eta d \xi+k_{2} \int^{x} \frac{d \xi}{\sigma_{1}(\xi)}+k_{3}
$$

in which $k_{1}, k_{2}$, and $k_{3}$ are constants of integration. The result for $\Psi(y)$ is

$$
\Psi(y)=-\int^{\nu} \frac{1}{\tau_{2}(\xi)} \int^{\xi}\left[k_{1} \tau_{1}(\eta)+\tau_{3}(\eta)\right] \Psi(\eta) d \eta d \xi+\int^{\nu} \frac{m_{1} d \xi}{\tau_{2}(\xi)}+m_{2},
$$

in which $m_{1}$ and $m_{2}$ are constants of integration. If, however, $\tau_{3}(y)=\tau_{1}(y)$, the expression for $\Phi(x)$ is of the form (3.11) and that for $\Psi(y)$ of the form (3.10).

In Case 2, the fact that we have

$$
\frac{\sigma_{2}(x)}{\sigma_{3}(x)}\left\{\frac{\tau_{2}(y) \Psi^{\prime \prime}}{\tau_{3}(y) \Psi}+\frac{\tau_{2}^{\prime}(y) \Psi^{\prime}}{\tau_{3}(y) \Psi}\right\}=\text { const. }
$$

implies that

$$
\frac{\sigma_{2}(x)}{\sigma_{3}(x)}=\text { const. } \quad \text { and } \frac{\tau_{2}(y) \Psi^{\prime \prime}}{\tau_{3}(y) \Psi}+\frac{\tau_{2}^{\prime}(y) \Psi^{\prime}}{\tau_{3}(y) \Psi}=\text { const. }=n_{1} .
$$

This yields an expression for $\Psi(y)$ of the form of (3.10). If we suppose $\sigma_{2}(x)=\sigma_{3}(x)$, equation (3.6) becomes

$$
\frac{d}{d z}\left\{n_{1} \frac{\pi_{2}(z)}{\pi_{1}(z)}+\frac{\pi_{3}(z) \mathrm{X}^{\prime \prime}}{\pi_{1}(z) \mathrm{X}}+\frac{\pi_{8}^{\prime}(z) \mathrm{X}^{\prime}}{\pi_{1}(z) \mathrm{X}}\right\}=0
$$

and the solution for $\mathbf{X}(z)$ is of the form of (3.11). Finally (3.5) is reduced to

$$
\frac{\tau_{1}(y)}{\tau_{3}(y) \sigma_{3}(x) \Phi} \frac{d}{d x}\left(\sigma_{1} \Phi^{\prime}\right)=\text { const. }
$$

This implies that

$$
\tau_{1}(y) / \tau_{3}(y)=\text { const., }
$$

completing the proof.

We note that in Case 2 the expression for $\Phi(x)$ is that given by (3.10).

The result obtained in Theorem (3.3) carries over directly to second order equations in the $n$ variables $x_{1}, x_{2}, \cdots, x_{n}$. Consider the equation 


$$
\sum_{i=1}^{n}\left\{\sum_{j=1}^{n} \alpha_{i j}\left(x_{j}\right) u_{x_{i}}\right\}_{x_{i}}=0,
$$

characterized by the matrix

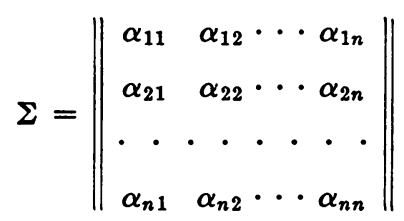

in which $\alpha_{i j}, i, j=1,2, \cdots, n$, is a function of the variables $x_{j}$. We assume that $\alpha_{i j}$ is analytic for all $i, j\left({ }^{7}\right)$.

(3.14) Definition. Suppose that in the $j$ th column of matrix (3.13) all the elements $\alpha_{i j}$ except $\alpha_{j j}$ are proportional. Then the matrix is said to satisfy Condition A in the jth column.

(3.15) TheOREM. A necessary and sufficient condition that (3.12) admit a nontrivial solution $u$ of the form

$$
u\left(x_{1}, x_{2}, \cdots, x_{n}\right)=\prod_{i=1}^{n} \Phi_{i}\left(x_{i}\right)
$$

is that every column of (3.13) except at most one satisfy Condition A.

The proof follows the same method as the proof of Theorem (3.3).

4. Generalized powers. As stated in Theorem (3.3) the equation

$$
\begin{aligned}
L u= & \left\{\sigma_{1}(x) \tau_{1}(y) \pi_{1}(z) u_{x}\right\}_{x}+\left\{\sigma_{2}(x) \tau_{2}(y) \pi_{2}(z) u_{y}\right\}_{y} \\
& +\left\{\sigma_{2}(x) \tau_{1}(y) \pi_{3}(z) u_{z}\right\}_{z}=0
\end{aligned}
$$

admits a solution of the form $u=\Phi(x) \Psi(y) \mathrm{X}(z)$. It will be assumed that the coefficients, characterized by the matrix

$$
\Sigma_{3}=\left\|\begin{array}{lll}
\sigma_{1}(x) & \tau_{1}(y) & \pi_{1}(z) \\
\sigma_{2}(x) & \tau_{2}(y) & \pi_{2}(z) \\
\sigma_{2}(x) & \tau_{1}(y) & \pi_{3}(z)
\end{array}\right\|
$$

are analytic $\left({ }^{8}\right)$ functions of their respective variables. Further, it will be assumed that the functions of (4.2) are positive so that equation (4.1) is of elliptic type.

A formal solution of (4.1) can be obtained from the expressions for $\Phi(x)$, $\Psi(y)$, and $X(z)$ given by (3.10), (3.11), and (3.8), respectively. We accom-

(7) These conditions can be weakened.

(8) For the definition of the powers and of the generalized spherical harmonics, it is sufficient that the coefficients of equation (4.1) have, for example, continuous first derivatives. 
plish this by substituting the initial conditions and then applying a process of iteration to each of the integral equations (3.10), (3.11), and (3.8). The initial conditions $\Phi(0)=1, \Phi^{\prime}(0)=0$ transform (3.10) into

$$
\Phi(x)=1+c_{1}^{2} \int_{0}^{x} \frac{1}{\sigma_{1}\left(\xi_{2}\right)} \int_{0}^{\xi_{2}} \sigma_{2}\left(\xi_{1}\right) \Phi\left(\xi_{1}\right) d \xi_{1} d \xi_{2}
$$

in which for convenience the constant $k_{1}$ has been replaced by $c_{1}^{2}$. We solve this equation by the method of successive approximation; we have

$$
\begin{aligned}
\Phi(x)=1 & +c_{1}^{2} \int_{0}^{x} \frac{1}{\sigma_{1}\left(\xi_{2}\right)} \int_{0}^{\xi_{2}} \sigma_{2}\left(\xi_{1}\right) d \xi_{1} d \xi_{2} \\
& +c_{1}^{4} \int_{0}^{x} \frac{1}{\sigma_{1}\left(\xi_{4}\right)} \int_{0}^{\xi_{4}} \sigma_{2}\left(\xi_{3}\right) \int_{0}^{\xi_{3}} \frac{1}{\sigma_{1}\left(\xi_{2}\right)} \int_{0}^{\xi_{2}} \sigma_{2}\left(\xi_{1}\right) d \xi_{1} \cdots d \xi_{4} \\
& +\cdots
\end{aligned}
$$

We define the function $X^{(2 n)}(x)$ by the rule

$$
\begin{aligned}
X^{(2 n)}(x)=(2 n) ! \int_{0}^{x} \frac{1}{\sigma_{1}\left(\xi_{1}\right)} & \int_{0}^{\xi_{1}} \sigma_{2}\left(\xi_{2}\right) \cdots \\
& \int_{0}^{\xi_{2 n-2}} \frac{1}{\sigma_{1}\left(\xi_{2 n-1}\right)} \int_{0}^{\xi_{2 n-1}} \sigma_{2}\left(\xi_{2 n}\right) d \xi_{1} \cdots d \xi_{2 n} .
\end{aligned}
$$

Then the expression $\Phi(x)$ takes the compact form

$$
\Phi(x)=\sum_{n=0}^{\infty} \frac{c_{1}^{2 n} X^{(2 n)}(x)}{(2 n) !} .
$$

The convergence of (4.4) follows from the inequality

$$
X^{(2 n)}(x) \leqq\left[\max _{|| \mid \leq x}\left\{\sigma_{2}(t), \frac{1}{\sigma_{1}(t)}\right\}\right]^{2 n} x^{2 n}=M^{2 n} x^{2 n}
$$

Thus (4.4) is dominated by the convergent series

$$
\sum_{n=0}^{\infty} \frac{\left\{c_{1} M(x) \cdot x\right\}^{2 n}}{(2 n) !} .
$$

The series for $\Phi^{\prime}(x)$ and $\left(1 / \sigma_{2}\right)(d / d x)\left[\sigma_{1} \Phi^{\prime}(x)\right]$ converge from similar considerations.

If the initial conditions $\Phi(0)=0, \Phi^{\prime}(0)=c_{1} / \sigma_{1}(0)$ are inserted in (3.10), the series for $\Phi(x)$ becomes

$$
\Phi(x)=c_{1} \int_{0}^{x} \frac{d \xi_{1}}{\sigma_{1}\left(\xi_{1}\right)}+c_{1}^{3} \int_{0}^{x} \frac{1}{\sigma_{1}} \int_{0}^{\xi_{1}} \sigma_{2} \int_{0}^{\xi_{2}} \frac{1}{\sigma_{1}} d \xi_{1} \cdots d \xi_{3}+\cdots .
$$


If the definition

$$
X^{(2 n+1)}(x)
$$

$$
=(2 n+1) ! \int_{0}^{x} \frac{1}{\sigma_{1}} \int_{0}^{\xi_{1}} \sigma_{2} \int_{0}^{\xi_{2}} \frac{1}{\sigma_{1}} \cdots \int_{0}^{\xi_{2 n-1}} \sigma_{2} \int_{0}^{\xi_{2 n}} \frac{1}{\sigma_{1}} d \xi_{1} \cdots d \xi_{2 n+1}
$$

is made, the expansion for $\Phi(x)$ takes the form

$$
\Phi(x)=\sum_{n=0}^{\infty} \frac{c_{1}^{2 n+1} X^{(2 n+1)}(x)}{(2 n+1) !} .
$$

The functions $X^{(n)}(x), n=0,1,2, \cdots$, will be called generalized powers.

In a similar way, the iteration process applied to expression (3.11) for $\Psi(y)$, first with the initial conditions $\Psi(0)=1, \Psi^{\prime}(0)=0$ and then with the initial conditions $\Psi(0)=0, \Psi^{\prime}(0)=c_{2} / \tau_{2}(0)$, leads to the definition of the generalized powers in $y$. Thus we have

$$
Y^{(2 n)}(y)=(2 n !) \int_{0}^{y} \frac{1}{\tau_{2}} \int_{0}^{\eta_{1}} \tau_{1} \int_{0}^{\eta_{2}} \frac{1}{\tau_{2}} \cdots \int_{0}^{\eta_{2 n-2}} \frac{1}{\tau_{2}} \int_{0}^{\eta_{2 n-1}} \tau_{1} d \eta_{1} \cdots d \eta_{2 n},
$$

$$
Y^{(2 n+1)}(y)=(2 n+1) ! \int_{0}^{\nu} \frac{1}{\tau_{2}} \int_{0}^{\eta_{1}} \tau_{1} \cdots \int_{0}^{\eta_{2 n-1}} \tau_{1} \int_{0}^{\eta_{2 n}} \frac{1}{\tau_{2}} d \eta_{1} \cdots d \eta_{2 n+1} .
$$

Then the two expansions for $\Psi(y)$ become

$$
\Psi(y)=\sum_{n=0}^{\infty} \frac{c_{2}^{2 n} Y^{(2 n)}}{(2 n) !}, \quad \Psi(y)=\sum_{n=0}^{\infty} \frac{c_{2}^{2 n+1} Y^{(2 n+1)}}{(2 n+1) !} .
$$

The convergence of these series follows from the same reasoning employed to show the convergence of (4.4).

To obtain the generalized powers of $z$ the process of iteration must be applied to the solution

$$
\mathrm{X}(z)=k_{1}+k_{2} \int_{0}^{z} \frac{d \zeta}{\pi_{3}(\zeta)}-\int_{0}^{z} \frac{1}{\pi_{3}\left(\zeta_{1}\right)} \int_{0}^{\zeta_{1}}\left\{c_{1}^{2} \pi_{1}\left(\zeta_{2}\right)+c_{2}^{2} \pi_{2}\left(\zeta_{2}\right)\right\} \mathrm{X}\left(\zeta_{2}\right) d \zeta_{1} d \zeta_{2}
$$

subject first to the initial conditions $X(0)=1, X^{\prime}(0)=0$ and then to the conditions $\mathbf{X}(0)=0, \mathbf{X}^{\prime}(0)=\left(c_{1}^{2}+c_{2}^{2}\right)^{1 / 2} / \pi_{3}(0)$. For the conditions $\mathbf{X}(0)=1, \mathbf{X}^{\prime}(0)=0$,

$$
\begin{aligned}
\mathrm{X}(z)=1 & -\int_{0}^{z} \frac{1}{\pi_{3}} \int_{0}^{\zeta_{1}}\left\{c_{1}^{2} \pi_{1}+c_{2}^{2} \pi_{2}\right\} d \zeta_{1} d \zeta_{2} \\
& +\int_{0}^{z} \frac{1}{\pi_{3}} \int_{0}^{\zeta_{1}}\left\{c_{1}^{2} \pi_{1}+c_{2}^{2} \pi_{2}\right\} \int_{0}^{\zeta_{2}} \frac{1}{\pi_{3}} \int_{0}^{\zeta_{3}}\left\{c_{1}^{2} \pi_{1}+c_{2}^{2} \pi_{2}\right\} d \zeta_{1} \cdots d \zeta_{4} \\
& +\cdots
\end{aligned}
$$


and hence

$$
\begin{aligned}
\mathrm{X}(z)=1 & -c_{1}^{2} \int_{0}^{2} \frac{1}{\pi_{3}} \int_{0}^{\zeta_{1}} \pi_{1} d \zeta_{1} d \zeta_{2}-c_{2}^{2} \int_{0}^{z} \frac{1}{\pi_{3}} \int_{0}^{\zeta_{1}} \pi_{2} d \zeta_{1} d \zeta_{2} \\
& +c_{1}^{4} \int_{0}^{z} \frac{1}{\pi_{3}} \int_{0}^{\zeta_{1}} \pi_{1} \int_{0}^{\zeta_{2}} \frac{1}{\pi_{3}} \int_{0}^{\zeta_{3}} \pi_{1} d \zeta_{1} \cdots d \zeta_{4} \\
& +c_{1}^{2} c_{2}^{2}\left\{\int_{0}^{z} \frac{1}{\pi_{3}} \int_{0}^{\zeta_{1}} \pi_{1} \int_{0}^{\zeta_{2}} \frac{1}{\pi_{3}} \int_{0}^{\zeta_{3}} \pi_{2} d \zeta_{1} \cdots d \zeta_{4}\right. \\
& \left.+\int_{0}^{2} \frac{1}{\pi_{3}} \int_{0}^{\zeta_{1}} \pi_{2} \int_{0}^{\zeta_{2}} \frac{1}{\pi_{3}} \int_{0}^{\zeta_{3}} \pi_{1} d \zeta_{1} \cdots d \zeta_{4}\right\}-+\cdots
\end{aligned}
$$

(4.8) Definition. The generalized power

$$
Z^{(2 m, 2 n)}(z)
$$

is the coefficient of $c_{1}^{2 m} c_{2}^{2 n}$ in the expansion (4.7).

Then the expression for $X(z)$ is

$$
\mathrm{X}(z)=\sum_{m, n=0}^{\infty}(-1)^{m+n} \frac{c_{1}^{2 m} c_{2}^{2 n} Z^{(2 m, 2 n)}(z)}{(2 m+2 n) !} .
$$

It will be useful to obtain explicit expressions for the powers $Z^{(2 m, 2 n)}(z)$. If one of the indices is zero, the generalized powers of $z$ are

$$
\begin{aligned}
& Z^{(2 m, 0)}(z)=(2 m) ! \int_{0}^{z} \frac{1}{\pi_{3}} \int_{0}^{\zeta_{1}} \pi_{1} \int_{0}^{\zeta_{2}} \frac{1}{\pi_{3}} \cdots \int_{0}^{\zeta 2 m-2} \frac{1}{\pi_{3}} \int_{0}^{\zeta 2 m-1} \pi_{1} d \zeta_{1} \cdots d \zeta_{2 m}, \\
& Z^{(0,2 n)}(z)=(2 n) ! \int_{0}^{z} \frac{1}{\pi_{3}} \int_{0}^{\zeta_{1}} \pi_{2} \int_{0}^{\zeta_{2}} \frac{1}{\pi_{3}} \cdots \int_{0}^{\zeta_{2 n-2}} \frac{1}{\pi_{3}} \int_{0}^{\zeta 2 n-1} \pi_{2} d \zeta_{1} \cdots d \zeta_{2 n} .
\end{aligned}
$$

We note that these are similar to the powers $X^{(2 m)}, Y^{(2 n)}$. For the general case, we define the quantities

$$
A=\int_{0}^{2} \frac{1}{\pi_{3}} \int_{0}^{\zeta_{1}} \pi_{1} d \zeta_{1} d \zeta_{2}, \quad B=\int_{0}^{2} \frac{1}{\pi_{3}} \int_{0}^{\zeta_{1}} \pi_{2} d \zeta_{1} d \zeta_{2},
$$

and we make the convention that a symbol of the form $A A B A B B \cdots A$, which consists of a finite product of $A$ 's and $B$ 's arranged in a particular order, shall represent the iterated integral of the $A$ 's and $B$ 's (and not the product of the integrals obtained by integrating the $A$ 's and $B$ 's separately).

(4.9) Lemma. The generalized powers $Z^{(2 m, 2 n)}(z)$ are given by the formula:

$$
\begin{aligned}
& Z^{(2 m, 2 n)}(z)=(2 m+2 n) ![\text { the sum of the }(m+n) ! /(m ! n !) \text { terms, } \\
& \text { consisting of all the distinct permutations of } \left.m \text { A's and } n B^{\prime} s\right] .
\end{aligned}
$$


Proof. We proceed by induction. The result holds for the cases $n=0$, $m=0,1,2, \cdots$, and for the cases $m=0, n=0,1,2, \cdots$. This follows at once from the explicit expressions given for $Z^{(2 m, 0)}(z)$ and $Z^{(0,2 n)}(z)$. The induction will be complete if the result is shown to hold for $Z^{(2 m+2,2 n)}(z)$ on the assumption that it holds for $Z^{(2 m, 2 n)}(z)$ and $Z^{(2 m+2,2 n-2)}(z)$. The quantity $Z^{(2 m+2,2 n)}(z)$ is obtained first by adjoining at the right end of each of the terms of the expression for $Z^{(2 m, 2 n)}(z)$ the element $A$, and then by adjoining the element $B$ to the right end of the terms of $Z^{(2 m+2,2 n-2)}(z)$. Hence there are

$$
\frac{(m+n) !}{m ! n !}+\frac{(m+n) !}{(m+1) !(n-1) !}=\frac{(m+n+1) !}{(m+1) ! n !}
$$

terms in $Z^{(2 m+2,2 n)}(z)$, each of which is a different one of the permutations of $m+1 A$ 's and $n B$ 's.

To obtain the generalized odd powers of $z$, the initial conditions

$$
\mathrm{X}(0)=0, \quad \mathrm{X}^{\prime}(0)=\frac{\left(c_{1}^{2}+c_{2}^{2}\right)^{1 / 2}}{\pi_{3}(0)}
$$

are inserted in the integral equation for $\mathbf{X}(z)$. The expansion becomes

$$
\begin{aligned}
\mathrm{X}(z)=\left(c_{1}^{2}+c_{2}^{2}\right)^{1 / 2}\{ & \int_{0}^{z} \frac{d \zeta_{1}}{\pi_{3}\left(\zeta_{1}\right)} \\
& \left.-\int_{0}^{z} \frac{1}{\pi_{3}} \int_{0}^{\zeta_{1}}\left(c_{1}^{2} \pi_{1}+c_{2}^{2} \pi_{2}\right) \int_{0}^{\zeta_{2}} \frac{1}{\pi_{3}} d \zeta_{1} d \zeta_{2} d \zeta_{3}+\cdots\right\} .
\end{aligned}
$$

Then the odd powers of $Z$ are obtained by equating the coefficients of $c_{1}$ and $c_{2}$ in the above series with the coefficients of $c_{1}$ and $c_{2}$ in the series

$$
\mathrm{X}(z)=\left(c_{1}^{2}+c_{2}^{2}\right)^{1 / 2} \sum_{m, n=0}^{\infty} \frac{(-1)^{m+n} c_{1}^{2 m} c_{2}^{2 n} Z^{(2 m, 2 n)+1}}{(2 m+2 n+1) !} .
$$

It is easily seen that the explicit expression for any odd power, $Z^{(2 m, 2 n)+1}(z)$, is obtained by adjoining the element

$$
\int_{0}^{\zeta_{1}} \frac{d \zeta_{2}}{\pi_{3}\left(\zeta_{2}\right)}
$$

to the right end of each of the terms that make up $Z^{(2 m, 2 n)}(z)$.

(4.11) Lemma. The generalized powers of $Z$ satisfy the recursion formula

$$
\begin{aligned}
& \frac{d}{d z}\left\{\pi_{3}(z) \frac{d}{d z} Z^{(2 m, 2 n)}(z)\right\} \\
& \quad=(2 m+2 n)(2 m+2 n-1)\left\{\pi_{1}(z) Z^{(2 m-2,2 n)}(z)+\pi_{2}(z) Z^{(2 m, 2 n-2)}(z)\right\} .
\end{aligned}
$$


Proof. We proceed by induction. The result clearly holds for the cases $n=0, m=0,1,2, \cdots$, and for the cases $m=0, n=0,1,2, \cdots$. This follows from the simple integral expressions for $Z^{(2 m, 0)}(z)$ and $Z^{(0,2 n)}(z)$. The induction will be complete if the result is shown to hold for $Z^{(2 m+2,2 n)}(z)$ on the assumption that it holds for $Z^{(2 m, 2 n)}(z)$ and $Z^{(2 m+2,2 n-2)}(z)$. From the explicit expressions obtained in Lemma $(4.9), Z^{(2 m+2,2 n)}(z)$ may be written symbolically $Z^{(2 m+2,2 n)}(z)=(2 m+2 n+2)(2 m+2 n+1)\left[Z^{(2 m, 2 n)}(z) A+Z^{(2 m+2,2 n-2)}(z) B\right]$.

Then we have

$$
\begin{aligned}
& \frac{d}{d z}\left\{\pi_{3}(z) \frac{d}{d z} Z^{(2 m+2,2 n)}(z)\right\} \\
& =(2 m+2 n+2)(2 m+2 n+1)\left\{( 2 m + 2 n ) ( 2 m + 2 n - 1 ) \left[\pi_{1}(z) Z^{(2 m-2,2 n)}(z) A\right.\right. \\
& \left.\left.\quad+\pi_{2}(z) Z^{(2 m, 2 n-2)}(z) A+\pi_{1}(z) Z^{(2 m, 2 n-2)}(z) B+\pi_{2}(z) Z^{(2 m+2,2 n-4)}(z) B\right]\right\},
\end{aligned}
$$

and again employing Lemma (4.9), we obtain

$$
\begin{aligned}
\frac{d}{d z}\left\{\pi_{3}(z)\right. & \left.\frac{d}{d z} Z^{(2 m+2,2 n)}(z)\right\} \\
& =(2 m+2 n+2)(2 m+2 n+1)\left[\pi_{1} Z^{(2 m, 2 n)}(z)+\pi_{2} Z^{(2 m+2,2 n-2)}(z)\right] .
\end{aligned}
$$

We note that the same formula and same argument apply to odd powers of $Z$.

5. Generalized spherical harmonics. It was seen in $\$ 2$ that the polynomials $R_{n, v}(x, y, z)$ form a class of solutions of the Laplace equation $\Delta u=0$. A corresponding class of solutions of equation (4.1), $L u=0$, is obtained by the substitution of generalized powers for ordinary powers in the polynomials $R_{n, y}(x, y, z)$. The method for replacement consists first of the substitution of the generalized powers $X^{(m)}$ and $Y^{(n)}$ for $x^{m}$ and $y^{n}$, respectively; then the variable $z^{2 N-2 r-2 s}$ is replaced by

$$
\frac{(\nu-r) !(N-\nu-s) !}{(N-r-s) !} Z^{(2 \nu-2 r, 2 N-2 \nu-2 s)}(z) .
$$

Thus a term $x^{2 r} y^{2 s} z^{2 N-2 r-2 s}$ corresponds to

$$
\frac{(\nu-r) !(N-\nu-s) !}{(N-r-s) !} X^{(2 r)}(x) Y^{(2 s)}(y) Z^{(2 \nu-2 r, 2 N-2 \nu-2 s)}(z) .
$$

With these replacement the polynomial $R_{n, \nu}(x, y, z)$ becomes the function $\bar{R}_{n, v}(X, Y, Z)$, a "polynomial" in the variables $X, Y, Z$.

(5.1) Definition. The functions $\bar{R}_{n, \nu}(X, Y, Z)$ are called generalized spherical harmonics.

(5.2) THEOREM. The generalized harmonics $\bar{R}_{n, \nu}(X, Y, Z)$ are solutions of equation (4.1) for all $n$ and $\nu$. 
Proof. Consider, for example, the harmonic polynomial

$$
R_{2 N, 4 \nu}(x, y, z)=\sum_{s=0}^{N-\nu} \sum_{r=0}^{\nu} \frac{(-1)^{N-r-s}(2 N) !(N-r-s) ! x^{2 r} y^{2 s} z^{2 N-2 r-2 s}}{(2 r) !(2 s) !(2 N-2 r-2 s) !(\nu-r) !(N-\nu-s) !}
$$

which transforms into the generalized harmonic

$$
\bar{R}_{2 N, 4 \nu}(X, Y, Z)=\sum_{s=0}^{N-\nu} \sum_{r=0}^{\nu} \frac{(-1)^{N-r-s}(2 N) ! X^{(2 r)} Y^{(2 s)} Z^{(2 p-2 r, 2 N-2 \nu-2 s)}}{(2 r) !(2 s) !(2 N-2 r-2 s) !} .
$$

Direct substitution of $\bar{R}_{2 N, 4 v}(X, Y, Z)$ into equation (4.1) yields

$$
\begin{aligned}
\sum_{r=0}^{N-\nu} \sum_{r=1}^{\nu} & \frac{(-1)^{N-r-s}(2 N) ! X^{(2 r-2)} Y^{(2 s)} Z^{(2 v-2 r, 2 N-2 v-2 s)} \sigma_{2}(x) \tau_{1}(y) \pi_{1}(z)}{(2 r-2) !(2 s) !(2 N-2 r-2 s) !} \\
& +\sum_{s=1}^{N-p} \sum_{r=0}^{\nu} \frac{(-1)^{N-r-s}(2 N) ! X^{(2 r)} Y^{(2 s-2)} Z^{(2 v-2 r, 2 N-2 v-2 s)} \sigma_{2}(x) \tau_{1}(y) \pi_{2}(z)}{(2 r) !(2 s-2) !(2 N-2 r-2 s) !} \\
& +\sum_{s=0}^{N-\nu} \sum_{r=0}^{\nu-1} \frac{(-1)^{N-r-s}(2 N) ! X^{(2 r)} Y^{(2 s)} Z^{(2 v-2 r-2,2 N-2 v-2 s)} \sigma_{2}(x) \tau_{1}(y) \pi_{1}(z)}{(2 r) !(2 s) !(2 N-2 r-2 s-2) !} \\
& +\sum_{s=0}^{N-\nu-1} \sum_{r=0}^{\nu} \frac{(-1)^{N-r-s}(2 N) ! X^{(2 r)} Y^{(2 s)} Z^{(2 v-2 r, 2 N-2 \nu-2 s-2)} \sigma_{2}(x) \tau_{1}(y) \pi_{2}(z)}{(2 r) !(2 s) !(2 N-2 r-2 s-2) !}
\end{aligned}
$$

It is seen by inspection that the first and third terms cancel and that the second and fourth terms cancel. In a similar way, the $\bar{R}_{n, \nu}(X, Y, Z)$ for other values of $n$ and $\nu$ may be shown to satisfy the equation.

6. Expansion theorem. It is the purpose of this section to show that every solution of equation (4.1) regular in the neighborhood of the origin can be expanded uniquely in a series

$$
u(x, y, z)=\sum_{n=0}^{\infty} \sum_{\nu=0}^{2 n} \frac{\beta_{n, \nu}}{n !} \bar{R}_{n, \nu}(X, Y, Z)
$$

provided that the coefficients of (4.1) are analytic functions of their respective variables. It is convenient to introduce the function

$$
m(r)=\max _{i=1,2} \max _{j=1,2,3} \max _{k=1,-1} \max _{|t| \leqq r}\left\{\sigma_{i}^{k}(t), \tau_{i}^{k}(t), \pi_{j}^{k}(t)\right\} .
$$

From the definitions of $X^{(n)}, Y^{(n)}$, and $Z^{(m, n)}$ given by (4.3), (4.6), and (4.8), respectively, it follows at once that

$$
\begin{aligned}
\left|X^{(n)}(x)\right| & \leqq m^{n}(x)|x|^{n} \\
\left|Y^{(n)}(y)\right| & \leqq m^{n}(y)|y|^{n} \\
\left|Z^{(2 v-2 r, 2 N-2 v-2 s)}(z)\right| & \leqq \frac{(N-r-s) !}{(\nu-r) !(N-\nu-s) !} m^{2 N-2 r-2 s}|z|^{2 N-2 r-2 s}
\end{aligned}
$$


(6.3) Theorem. If the series

$$
\sum_{n=0}^{\infty}\left|\sum_{\nu=0}^{2 n} \frac{\beta_{n, \nu}}{n !} R_{n, \nu}(x, y, z)\right|
$$

converges in a neighborhood of the origin, say for

$$
|x|+|y|+|z| \leqq r_{0},
$$

then the series

$$
u(x, y, z)=\sum_{n=0}^{\infty} \sum_{\nu=0}^{2 n} \frac{\beta_{n, v}}{n !} \bar{R}_{n, v}(X, Y, Z)
$$

converges absolutely and uniformly in a sufficiently small neighborhood of the origin. Further, the function $u(x, y, z)$ is a solution of equation (4.1), and the series (6.5) can be differentiated term by term with respect to the variables $x, y$, and $z$.

Proof. From the inequalities (6.2) we have

$$
\sum_{n=0}^{\infty} \sum_{r=0}^{2 n} \frac{\left|\beta_{n, \nu}\right|}{n !}\left|\bar{R}_{n, \nu}(X, Y, Z)\right| \leqq \sum_{n=0}^{\infty} \sum_{n=0}^{2 n} M^{n} \frac{\left|\beta_{n, \nu}\right|}{n !}\left|R_{n, \nu}(x, y, z)\right| .
$$

It is clear that (6.4) represents a harmonic function and hence Theorem (2.15) may be applied. Thus the series

$$
\sum_{n=0}^{\infty} \sum_{\nu=0}^{2 n} M^{n} \frac{\left|\beta_{n, \nu}\right|}{n !}\left|R_{n, v}(x, y, z)\right|
$$

converges uniformly in the neighborhood $|x|+|y|+|z| \leqq r_{0} / 2 M$. Since (6.4) represents a harmonic function the above argument may be applied to the derived series of (6.4) and (6.5). Hence the series (6.5) may be differentiated term by term. If the series (6.5) is substituted in equation (4.1), we find

$$
\begin{aligned}
\sum_{n=0}^{\infty} \sum_{\nu=0}^{2 n} \frac{\beta_{n, \nu}}{n !}\left[\sigma_{1} \tau_{1} \pi_{1} \frac{\partial}{\partial x} \bar{R}_{n, \nu}\right]_{x} & {\left[\sigma_{1} \tau_{2} \pi_{2} \frac{\partial}{\partial y} \bar{R}_{n, \nu}\right]_{y} } \\
& +\left[\sigma_{2} \tau_{1} \pi_{3} \frac{\partial}{\partial z} \bar{R}_{n, \nu}\right]_{z}=0 .
\end{aligned}
$$

By Theorem (5.2), $\bar{R}_{n, v}(X, Y, Z)$ is a solution of (4.1); hence the function $u(x, y, z)$ given by the series $(6.5)$ is a solution of $(4.1)$.

The following lemma, stated without proof, is a simple generalization of a lemma given by Bers and Gelbart [4, Lemma 6.1].

(6.6) Lemma. Let $F\left(z_{1}, z_{2}\right), G_{1}\left(z_{1}\right), G_{2}\left(z_{2}\right), H_{1}\left(z_{1}\right), H_{2}\left(z_{2}\right)$, be analytic functions of $z_{1}$ and $z_{2}$ defined in the neighborhood of the origin. Let $F_{00}\left(z_{1}, z_{2}\right)=F\left(z_{1}, z_{2}\right)$ and define 


$$
F_{i j}\left(z_{1}, z_{2}\right)= \begin{cases}G_{1}\left(z_{1}\right) \frac{d}{d z_{1}} F_{i-1, j}\left(z_{1}, z_{2}\right), & i \text { odd, } \\ G_{2}\left(z_{2}\right) \frac{d}{d z_{2}} F_{i, j-1}\left(z_{1}, z_{2}\right), & j \text { odd }, \\ H_{1}\left(z_{1}\right) \frac{d}{d z_{1}} F_{i-1, j}\left(z_{1}, z_{2}\right), & i \text { even, } \\ H_{2}\left(z_{2}\right) \frac{d}{d z_{2}} F_{i, j-1}\left(z_{1}, z_{2}\right), & j \text { even. }\end{cases}
$$

Then there exists a positive constant $C$ such that

$$
\left|F_{i j}(0,0)\right| \leqq(i+j) ! C^{i+j} .
$$

In carrying over the analogy of the expansion of solutions of the equation $\Delta u=0$ to the equation $L u=0$, we find it convenient to introduce derivatives with respect to generalized powers. If we suppose $u=u[X(x), Y(y), Z(z)]$, then we define

$$
\frac{\partial u}{\partial X}=\sigma_{1}(x) \frac{\partial u}{\partial x}, \quad \frac{\partial^{2} u}{\partial x^{2}}=\frac{1}{\sigma_{2}(x) \cdot} \frac{\partial}{\partial x}\left(\sigma_{1}(x) \frac{\partial u}{\partial x}\right)
$$

and, in general,

$$
\frac{\partial^{2 n+1} u}{\partial X^{2 n+1}}=\sigma_{1}(x) \frac{\partial}{\partial x} \frac{\partial^{2 n} u}{\partial X^{2 n}}, \quad \frac{\partial^{2 n} u}{\partial X^{2 n}}=\frac{1}{\sigma_{2}(x)} \frac{\partial}{\partial x} \frac{\partial^{2 n-1} u}{\partial X^{2 n-1}} .
$$

The derivatives with respect to $Y$ are defined by the similar formulas

$$
\frac{\partial^{2 n+1} u}{\partial Y^{2 n+1}}=\tau_{2}(y) \frac{\partial}{\partial y} \frac{\partial^{2 n} u}{\partial Y^{2 n}}, \quad \frac{\partial^{2 n} u}{\partial Y^{2 n}}=\frac{1}{\tau_{1}(y)} \frac{\partial}{\partial y} \frac{\partial^{2 n-1} u}{\partial Y^{2 n-1}} .
$$

For the present development it is merely necessary to define the first derivative of $u$ with respect to $Z$. This becomes

$$
\frac{\partial u}{\partial Z}=\pi_{3}(z) \frac{\partial u}{\partial z} .
$$

Suppose $u(x, y, z)$ is an analytic function of $x, y$, and $z$ in the neighborhood of the origin. We shall employ the auxiliary functions.

$$
\begin{gathered}
u_{1}(x, y)=u(x, y, 0), \quad u_{2}(x, y)=\left.\frac{\partial}{\partial Z} u(x, y, z)\right|_{=-0}, \\
u_{3}(x, y)=u_{1}(x, y)+u_{2}(x, y) .
\end{gathered}
$$

We also define the quantities 


$$
\begin{gathered}
\beta_{n, 2 \nu}=\left.\frac{\nu !(n-\nu) !}{n !} \frac{\partial^{n} u_{1}(x, y)}{\partial X^{\nu} \partial Y^{n-\nu}}\right|_{x=\nu=0}, \\
\beta_{n, 2 v+1}=\left.\frac{\nu !(n-\nu-1) !}{(n-1) !} \frac{\partial^{n-1} u_{2}(x, y)}{\partial X^{\nu} \partial Y^{n-\nu-1}}\right|_{x=y=0} .
\end{gathered}
$$

(6.11) THEOREM. Let $u(x, y, z)$ satisfy equation (4.1), Lu=0, and be analytic in the neighborhood of the origin. Then, if the quantities $\beta_{n, \nu}$ given by (6.10) vanish for all $n$ and $\nu$, the function $u(x, y, z)$ vanishes identically.

Proof. From the definition of generalized derivatives with respect to $X$ and $Y$ and from the fact that $\tau_{2}(0) \neq 0$, it follows that

$$
\left.\frac{\partial u_{2}}{\partial y}\right|_{x=y=0}=0 \text {. }
$$

Similarly it follows that all derivatives of $u_{1}(x, y)$ and $u_{2}(x, y)$ vanish at the origin. Since $\beta_{0,0}=u_{1}(0,0)=0$ and $\beta_{1,1}=u_{2}(0,0)=0$, it follows that $u_{3}(x, y)$ vanishes identically. Then $u(x, y, z)$ vanishes identically in the $x y$-plane and so does its derivative with respect to $z$. Hence, by the Cauchy-Kowaleski theorem, the function $u(x, y, z)$ vanishes identically.

(6.12) THEOREM. Let $u(x, y, z)$ be a solution of equation (4.1), $L u=0$, regular in a neighborhood $D$ of the origin. Let the quantities $\beta_{n, v}$ be formed as in (6.10). Then the series

$$
\sum_{n=0}^{\infty} \sum_{\nu=0}^{2 n} \frac{\beta_{n, \nu}}{n !} \bar{R}_{n, \nu}(X, Y, Z)
$$

converges and represents $u(x, y, z)$ in some neighborhood of the origin contained in $D$.

Proof. Consider the series

$$
\sum_{n=0}^{\infty} \sum_{\nu=0}^{2 n} \frac{\beta_{n, v}}{n !} R_{n, \nu}(x, y, z) .
$$

From relations (6.10) and Lemma (6.6), we have

$$
\left|\beta_{n, 2 \nu}\right| \leqq \frac{\nu !(n-\nu) !}{n !} n ! C_{1}^{n}, \quad\left|\beta_{n, 2 v+1}\right| \leqq \frac{\nu !(n-\nu-1 \asymp}{(n-1) !}(n-1) ! C_{2}^{n-1} .
$$

For $|x|+|y|+|z| \leqq 2 \epsilon$ and for all $n$ and $\nu$, the relation $\left|R_{n, \nu}(x, y, z)\right| \leqq \epsilon^{n}$ holds. If we take into account the inequality $\nu !(n-\nu) ! \leqq n !$, then it follows that the series (6.16) is dominated by the series $\sum_{n=0}^{\infty}(C \epsilon)^{n}$. Hence, for $\epsilon<1 / C$, that is, in the domain $|x|+|y|+|z| \leqq 2 / C$, the series (6.14) converges. From Theorem (6.3) it follows that (6.13) converges in a sufficiently small 
neighborhood of the origin. By Theorem (6.11) the function $u$ is represented uniquely.

7. Binomial formulas for generalized spherical harmonics. The solutions of equation (4.1), $L u=0$, considered thus far have been those regular in the neighborhood of the origin. Suppose now that $L u=0$ has a solution $u(x, y, z)$ regular in the neighborhood of some point $P\left(x_{0}, y_{0}, z_{0}\right)$. The development in this case would consist first of defining the generalized powers $X^{(n)}, Y^{(n)}$, and $Z^{(m, n)}$ in the same way as was done in $\$ 4$. Here, however, the lower limits of the integrals defining $X^{(n)}$ are $x_{0}$ instead of zero. Similarly, for $Y^{(n)}$ and $Z^{(m, n)}$ the lower limits of the integrals would be $y_{0}$ and $z_{0}$, respectively. With these definitions of generalized powers, the generalized spherical harmonics $\bar{R}_{n, v}(X, Y, Z)$ can be formed in the same way as in $\S 5$. For these harmonics defined "with respect to the point $P$ " we introduce the notation $\bar{R}_{n, v}(X, Y, Z ; P)$.

Since $\bar{R}_{n, \nu}(X, Y, Z ; P)$ is a solution of $L u=0$ regular in the entire space, it can be expanded in terms of $\bar{R}_{n, v}(X, Y, Z ; 0)=\bar{R}_{n, v}(X, Y, Z)$. That is, the theorems of $\$ 6$ can be applied. Thus we have

$$
\bar{R}_{n, \nu}(X, Y, Z ; P)=\sum_{m=0}^{\infty} \sum_{\mu=0}^{2 m} \frac{\alpha_{m, \mu}}{m !} \bar{R}_{m, \mu}(X, Y, Z),
$$

which follows directly from the expansion theorem, (6.12). The coefficients $\alpha_{m, \mu}$ are given by the formulas (6.10) with $u(x, y, z)$ replaced by $\bar{R}_{n, v}(X, Y, Z ; P)$. From the definition of generalized harmonics it is clear that $\bar{R}_{n, \nu}(X, Y, Z ; P)$ contains no generalized power of degree greater than $n$. The generalized derivative of a generalized power follows the same rule as the ordinary derivative of an ordinary power. The formulas (6.10) for the coeffcients $\alpha_{m, \mu}$ consist essentially of the various $m$ th derivatives with respect to $X$ and $Y$ evaluated at the origin. Hence, since $\bar{R}_{n, \nu}(X, Y, Z ; P)$ is a "polynomial" in $X, Y$, and $Z$, all derivatives greater than the $n$th will be zero. Thus $\alpha_{m, \mu}=0$ for $m>n$. Then the series for $\bar{R}_{n, \nu}(X, Y, Z ; P)$ is

$$
\bar{R}_{n, \nu}(X, Y, Z ; P)=\sum_{m=0}^{n} \sum_{\mu=0}^{2 m} \frac{\alpha_{m, \mu}}{m !} \bar{R}_{m, \mu}(X, Y, Z)
$$

in which

$$
\begin{gathered}
\alpha_{m, 2 \mu}=\left.\frac{\mu !(m-\mu) !}{m !} \cdot \frac{\partial^{m} \bar{R}_{n, \nu}(X, Y, 0 ; P)}{\partial X^{\mu} \partial Y^{m-\mu}}\right|_{x=y-0}, \\
\alpha_{m, 2 \mu+1}=\left.\frac{\mu !(m-\mu-1) !}{(m-1) !}\left[\frac{\partial^{m-1}}{\partial X^{\mu} \partial Y^{m-\mu-1}}\left\{\left.\frac{\partial}{\partial Z} \bar{R}_{n, \nu}(X, Y, Z ; P)\right|_{z=0}\right\}\right]\right|_{x=y-0} .
\end{gathered}
$$

By the results of $\S 6$ a solution $u(x, y, z)$ of $L u=0$ regular in the neighborhood of a point $P\left(x_{0}, y_{0}, z_{0}\right)$ can be expanded in powers of $\bar{R}_{n, v}(X, Y, Z ; P)$. Thus we have 


$$
u(x, y, z)=\sum_{n=0}^{\infty} \sum_{v=0}^{2 n} \frac{\beta_{n, \nu}}{n !} \bar{R}_{n, \nu}(X, Y, Z ; P)
$$

in which the coefficients $\beta_{n, v}$ are given by the formulas

$$
\begin{aligned}
\beta_{n, 2 \nu} & =\left.\frac{\nu !(n-\nu) !}{n !} \frac{\partial^{n} u\left(x, y, z_{0}\right)}{\partial X^{\nu} \partial Y^{n-\nu}}\right|_{x=x_{0}, y-y_{0}}, \\
\beta_{n, 2 \nu+1} & =\left.\frac{\nu !(n-\nu-1) !}{(n-1) !} \frac{\partial^{n-1}}{\partial X^{\nu} \partial Y^{n-\nu-1}}\left\{\left.\frac{\partial}{\partial Z} u(x, y, z)\right|_{z=z_{0}}\right\}\right|_{z=x_{0}, y=y_{0}} .
\end{aligned}
$$

The expansions (7.3) and (7.1) yield the following theorem.

(7.5) THEOREM. Let $u(x, y, z)$ be a solution of $L u=0$ regular in the neighborhood of a point $P\left(x_{0}, y_{0}, z_{0}\right)$. Then $u(x, y, z)$ can be expanded in the series

$$
u(x, y, z)=\sum_{n=0}^{\infty} \sum_{\nu=0}^{2 n} \frac{\beta_{n, \nu}}{n !} \sum_{m=0}^{n} \sum_{\mu=0}^{2 m} \frac{\alpha_{m, \mu}}{m !} \bar{R}_{m, \mu}(X, Y, Z)
$$

in which the $\alpha_{m, \mu}$ and $\beta_{n, \nu}$ are given by (7.2) and (7.4), respectively. (The generalized harmonics $\bar{R}_{n, y}(X, Y, Z)$ are those taken with respect to the origin.)

8. Examples. If the spherical coordinates $x=r \sin \theta \cos \phi, y=r \sin \theta \sin \phi$, $z=r \cos \theta$ are introduced, the Laplace equation becomes

$$
\sin \theta \frac{\partial}{\partial r}\left(r^{2} \frac{\partial u}{\partial r}\right)+\frac{1}{\sin \theta} \frac{\partial^{2} u}{\partial \phi^{2}}+\frac{\partial}{\partial \theta}\left(\sin \theta \frac{\partial u}{\partial \theta}\right)=0 .
$$

As is well known, any solution of (8.1) can be expanded in the series

$$
\sum_{n=0}^{\infty} r^{n} \sum_{m=0}^{\infty}\left(A_{n}^{m} \cos m \phi+B_{n}^{m} \sin m \phi\right) P_{n}^{m}(\cos \theta)
$$

where the $A_{n}^{m}, B_{n}^{m}$ are constants and the $P_{n}^{m}(\cos \theta)$ are the associated Legendre functions. (8.1) is of the type of (4.1) with the coefficients forming the matrix

$$
\left\|\begin{array}{ccc}
r^{2} & 1 & \sin \theta \\
1 & 1 & 1 / \sin \theta \\
1 & 1 & \sin \theta
\end{array}\right\| .
$$

The generalized powers $X^{(n)}(r), Y^{(n)}(\phi)$, and $Z^{(m, n)}(\theta)$ can be formed, and any solution of (8.1) analytic in the neighborhood of a point $P$ can be expanded in a series

$$
\sum_{n=0}^{\infty} \sum_{\nu=0}^{2 n} \frac{\alpha_{n, \nu}}{n !} \bar{R}_{n, \nu}\{X(r), Y(\phi), Z(\theta)\}
$$

in which the coefficients are given by formulas (7.4). 
It is clear that the quantities

$$
r^{n} P_{n}^{m}(\cos \theta) \underset{\cos }{\sin } m \phi
$$

satisfy relations of the form

$$
r^{n} P_{n}^{m}(\cos \theta){ }_{\cos }^{\sin } m \phi=\sum_{k=0}^{n} \sum_{\nu=0}^{2 k} \frac{\alpha_{k, \nu}}{k !} R_{k, \nu}(x, y, z)
$$

where the coefficients $\alpha_{k, \nu}$ are given by (2.14). These coefficients can be evaluated with the aid of Ferrer's formula. (See [6, p. 392].) From this formula it follows that

$$
\int_{-\pi}^{\pi}(z+i x \cos v+i y \sin v)^{n}{ }_{\cos }^{\sin } m v d v=\frac{2 \pi i^{m} n !}{(m+n) !} r^{n} P_{n}^{m}(\cos \theta){ }_{\cos }^{\sin } m \phi .
$$

We introduce the quantities

$$
\begin{gathered}
e=\left[\frac{m+n+1}{2}\right]-\left[\frac{m+n}{2}\right], \quad f=\left[\frac{m+1}{2}\right]-\left[\frac{m}{2}\right], \\
g=\left[\frac{n+1}{2}\right]-\left[\frac{n}{2}\right]
\end{gathered}
$$

where $m=0,1,2, \cdots, n=0,1,2, \cdots$, and we let

$$
A_{m, n}=\frac{(-1)^{m+n+e}(m+n) ! m !}{n ! 2^{m+n-\bullet}[(m+n) / 2] !} .
$$

(8.4) THEOREM. Every solution of the Laplace equation of the form

$$
r^{n} P_{n}^{m}(\cos \theta) \cos _{\sin }^{\sin } m \phi
$$

can be expressed as a linear combination of the quantities $R_{n, v}(x, y, z)$ given by:

$$
r^{n} P_{n}^{m}(\cos \theta) \cos m \phi=A_{m, n} \sum_{\nu=0}^{[n / 2]} R_{n, 2(2 v+f)}
$$

$$
\begin{gathered}
\sum_{l=0}^{[m / 2]} \frac{(-1)^{l}(m+2 \nu-2 l+f) !(n-2 \nu+2 l-g) !}{(2 l) !(m-2 l) !((m+f) / 2+\nu-l) !((n-g) / 2-\nu+l) !} \text { (for } m+n \text { even) } \\
=A_{m, n} \sum_{\nu=0}^{[(n-1) / 2]} R_{n, 2(2 \nu+f)+1} \\
\sum_{l=0}^{[m / 2]} \frac{(-1)^{l}(m+2 \nu-2 l+f) !(n-2 \nu+2 l-1-g) !}{(2 l) !(m-2 l) !((m+f) / 2+\nu-l) !((n-g-1) / 2-\nu+l) !} \text { (for } m+n \text { odd); }
\end{gathered}
$$




$$
\begin{aligned}
& r_{n}^{n} P_{n}^{m}(\cos \theta) \sin m \phi=A_{m, n} \sum_{\nu=0}^{\lfloor(n-1) / 2]} R_{n, 2(2 \nu+1-g)} \\
& \sum_{l=0}^{[(m-1) / 2]} \frac{(-1)^{l}(m+2 \nu-2 l-f) !(n-2 \nu+2 l+g) !}{(2 l+1) !(m-2 l-1) !((m-f) / 2+\nu-l) !((n+g) / 2-\nu+l) !} \text { (for } m+n \text { even) } \\
& =A_{m, n} \sum_{\nu=0}^{[(n-1) / 2]} R_{n, 2(2 v+o)+1} \\
& \sum_{l=0}^{[(m-1) / 2]} \frac{(-1)^{l}(m+2 \nu-2 l-f) !(n-2 \nu+2 l-g) !}{(2 l+1) !(m-2 l-1) !((m-f) / 2+\nu-l) !((n+g) / 2-\nu+l) !} \text { (for } m+n \text { odd). }
\end{aligned}
$$

Proof. Multiplication of (8.3) by $i^{-n}$ yields

$$
\int_{-\pi}^{\pi}(x \cos v+y \sin v-i z)^{n} \cos m v d v=\frac{i^{m-n} 2 \pi n !}{(m+n) !} r^{n} P_{n}^{m}(\cos \theta) \cos m \phi,
$$

and a similar expression with $\sin (m \phi)$. On the other hand, the definition of the polynomials $R_{n, y}(x, y, z)$ as the coefficients in the expansion of the functions $e^{\alpha x} e^{\beta y} e^{i \gamma z}$ implies that

$$
\begin{aligned}
u=e^{\alpha x+\beta y+i \gamma z} & =\sum_{n=0}^{\infty} \frac{(\alpha x+\beta y+i \gamma z)^{n}}{n !} \\
& =\sum_{n=0}^{\infty} \sum_{\nu=0}^{n} \frac{\alpha^{\nu} \beta^{n-\nu}}{n !}\left[R_{n, 2 \nu}+\frac{i \gamma}{n+1} R_{n+1,2 v+1}\right] .
\end{aligned}
$$

Hence we have

$$
\operatorname{Re}(\alpha x+\beta y+i \gamma z)^{n}=\sum_{v=0}^{n} \alpha^{\nu} \beta^{n-\nu} R_{n, 2 v}
$$

and

$$
\operatorname{Im}(\alpha x+\beta y+i \gamma z)^{n}=\gamma \sum_{\nu=0}^{n-1} \alpha^{\nu} \beta^{n-\nu-1} R_{n, 2 \nu+1} .
$$

If the substitution $\alpha=\cos v, \beta=\sin v$ is made, the relation $\alpha^{2}+\beta^{2}=\gamma^{2}$ yields $\gamma= \pm 1$,

$$
\operatorname{Re}(x \cos v+y \sin v \pm i z)^{n}=\sum_{v=0}^{n} \cos ^{v} v \sin ^{n-v} v R_{n, 2 v}
$$

and

$$
\operatorname{Im}(x \cos v+y \sin v \pm i z)^{n}= \pm \sum_{v=0}^{n-1} \cos ^{\nu} v \sin ^{n-v-1} v R_{n, 2 v+1} .
$$

Multiplying by $\cos (m v)$ and integrating from $-\pi$ to $+\pi$, we find 


$$
\begin{aligned}
\int_{-\pi}^{\pi} \operatorname{Re}(x \cos v+y \sin v \pm i z)^{n} & \cos m v d v \\
& =\sum_{n=0}^{n} R_{n, 2 v} \int_{-\pi}^{\pi} \cos ^{\nu} v \sin ^{n-v} v \cos m v d v,
\end{aligned}
$$

and a similar expression for the imaginary part. For the case of $m$ and $n$ even relation ( 8.7 ) becomes, after integration,

$$
\begin{aligned}
& \int_{-\pi}^{\pi} \operatorname{Re}(x \cos v+y \sin v \pm i z)^{n} \cos m v d v \\
& \quad=\sum_{\nu=0}^{n / 2} \sum_{l=0}^{m / 2} R_{n, 4 \nu} \frac{(-1)^{l} m !(m-2 l+2 \nu) !(n+2 l-2 v) ! 2 \pi}{(2 l) !(m-2 l) !(m / 2-l+v) !(n / 2+l-\nu) !((m+n) / 2) ! 2^{m+n}} .
\end{aligned}
$$

For both $m$ and $n$ odd (8.7) yields a similar expression. A comparison of (8.8) with (8.6) yields, for both $m$ and $n$ even,

$r^{n} P_{n}^{m}(\cos \theta) \cos m \phi$

$$
=\frac{(-1)^{(m+n) / 2}(m+n) ! m !}{n !((m+n) / 2) ! 2^{m+n}} \sum_{\nu=0}^{n / 2} R_{n, 4 \nu} \sum_{l=0}^{m / 2} \frac{(-1)^{l}(m-2 l+2 \nu) !(n+2 l-2 \nu) !}{(2 l) !(m-2 l) !(m / 2-l+\nu) !(n / 2+l-\nu) !} .
$$

The remaining relations, (8.5), are found by a study of the various combinations of $m$ and $n$ even and odd.

If the cylindrical coordinates $x=v \cos \phi, y=v \sin \phi, z=w$ are introduced, the Laplace equation becomes

$$
v \frac{\partial^{2} u}{\partial w^{2}}+\frac{1}{v} \frac{\partial^{2} u}{\partial \phi^{2}}+\dot{v} \frac{\partial^{2} u}{\partial v^{2}}+\frac{\partial u}{\partial v}=0 .
$$

Equation (8.9) is in the form of (4.1) with the matrix

$$
\left\|\begin{array}{ccc}
1 & 1 & v \\
1 & 1 & 1 / v \\
1 & 1 & v
\end{array}\right\| .
$$

We consider the generalized spherical harmonics generated by equation (8.9) about the point $P_{0}: w=0, \phi=0, v=1$. In this case the powers $X^{(n)}(w)$ and $Y^{(n)}(\phi)$ are merely the ordinary powers $w^{n}$ and $\phi^{n}$, respectively. The powers $Z^{(m, n)}(v)$ can be formed according to the methods of $\S 4$. Since, as is well known, the function $\cosh (w) \cos (n \phi) J_{n}(v)$ is a solution of $(8.9)$, we have the expansion

$$
\cos (n w) \cos (n \phi) J_{n}(v)=\sum_{k=0}^{\infty} \sum_{\nu=0}^{2 k} \frac{\alpha_{k, \nu}}{k !} \bar{R}_{k, \nu}\{X(w), Y(\phi), Z(v)\}
$$


in which

$$
\begin{aligned}
\alpha_{2 k, 4 v} & =\frac{(2 \nu) !(2 k-2 v) !}{(2 k) !}(-1)^{k-v} n^{2 k-2 v} J_{n}(1), \\
\alpha_{2 k+1,4 v+1} & =\frac{(2 v) !(2 k-2 v) !}{(2 k) !}(-1)^{k-v} n^{2 k-2 v} J_{n}^{\prime}(1), \\
\alpha_{k, 4 v+2} & =\alpha_{k, 4 v+3}=\alpha_{2 k+1,4 v}=\alpha_{2 k, 4 v+1}=0 .
\end{aligned}
$$

Hence, the series (8.10) can be written

$\cosh w \cos (n \phi) J_{n}(v)$

$$
=\sum_{k=0}^{\infty} \sum_{\nu=0}^{k} \frac{(2 v) !(2 k-2 \nu) !}{(2 k) !}(-1)^{k-v} n^{2 k-2 \nu}\left[\frac{J_{n}(1)}{(2 k) !} \bar{R}_{2 k, 4 \nu}+\frac{J_{n}^{\prime}(1)}{(2 k+1) !} \bar{R}_{2 k+1,4 v+1}\right] \text {. }
$$

In particular, for $w=\phi=0$ we have the series expansion for the Bessel function $J_{n}(v)$

$$
\begin{aligned}
J_{n}(v)= & \sum_{k=0}^{\infty} \sum_{v=0}^{k} \frac{(2 \nu) !(2 k-2 \nu) !}{(2 k) !}(-1)^{2 k-v} n^{2 k-2 v} \\
& \cdot\left[\frac{J_{n}(1)}{(2 k) !} Z^{(2 v, 2 k-2 v)}+\frac{J_{n}^{\prime}(1)}{(2 k+1) !} Z^{(2 v, 2 k-2 v)+1}\right] .
\end{aligned}
$$

Each of the generalized powers of $Z$ in (8.11) consists of the sum of integrals of the type $\int x^{p}(\log x) d x$. Therefore each term of the series can be obtained in closed form.

When expressed in rectangular coordinates the quantities cosh $w$ $\cdot \cos (n \phi) J_{n}(v)$ are solutions of $\Delta u=0$. Hence, by the results of $\S 4$ we can obtain the expansion in the ordinary harmonics

$$
\cosh w \cos (n \phi) J_{n}(v)=\sum_{k=0}^{\infty} \sum_{\nu=0}^{2 k} \frac{\beta_{k, \nu}}{k !} R_{k, \nu}(x, y, z) .
$$

To obtain expressions for the Bessel functions in terms of the harmonics $R_{n, v}(x, y, z)$, that is, to evaluate the coefficients $\beta_{k, v}$ above, we make use of the formula (see $[6$, p. 396])

$$
\int_{-\pi}^{\pi} \exp (x \cos u+y \sin u-i w) \cos (m u) d u=2 \pi i^{m} e^{-i w} \cos (m \phi) J_{m}(-i v) \text {. }
$$

Then by an argument similar to the one employed in the proof of Theorem (8.4) we have

$i^{m} \cos w \cos (m \phi) J_{m}(-i v)$

$$
=\sum_{n=0}^{\infty} \sum_{\nu=0}^{n} \sum_{l=0}^{[m / 2]} \frac{(-1)^{l} m !(m-2 l+2 \nu+f) !(2 n+2 l-2 \nu) ! R_{2 .+\gamma, 2(2 \nu+\gamma)}(x, y, z)}{(2 l) !(m-2 l) !(2 n+f) !\{[(m+1) / 2]+\nu-l\} !(n+l-\nu) !\{[(m+1) / 2]+n\} ! 2^{m+2 n+\gamma}},
$$


$-i^{m} \sin w \cos (m \phi) J_{m}(-i v)$

$$
=\sum_{n=0}^{\infty} \sum_{\nu=0}^{n} \sum_{l=0}^{[m / 2]} \frac{(-1)^{l} m !(m-2 l+2 \nu+f) !(2 n+2 l-2 \nu) ! R_{2 n+f+1.2(2 \nu+f)+1}(x, y, z)}{(2 l) !(m-2 l) !(2 n+f+1) !\{[(m+1) / 2]+\nu-l\} !(n+l-\nu) !\{[(m+1) / 2]+n\} ! 2^{m+2 n+f}} \cdot
$$

In particular, for $w=\phi=0$, we get the expansion for the Bessel function $J_{m}(-i x)$

$$
\begin{aligned}
& (-1)^{[m / 2]} i^{f} J_{m}(-i x) \\
& =\frac{m !}{2^{m}} \sum_{n=0}^{\infty} \frac{x^{2 n+f}}{(2 n+f) !([(m+1) / 2]+n) ! 2^{2 n+f}} \sum_{l=0}^{[m / 2]} \frac{(-1)^{l}(m+2 n-2 l+f) !}{l !(m-2 l) !([(m+1) / 2]+n-l) !} .
\end{aligned}
$$

Stepanoff [5] has considered those Euclidean transformations of coordinates

$$
x=X\left(t_{1}, t_{2}, t_{3}\right), \quad y=Y\left(t_{1}, t_{2}, t_{3}\right), \quad z=Z\left(t_{1}, t_{2}, t_{3}\right)
$$

which transform the Laplace equation into an equation possessing solutions of the form $u=T_{1}\left(t_{1}\right) T_{2}\left(t_{2}\right) T_{8}\left(t_{3}\right)$. It is shown that there are essentially five such transformations; in each case the transformed equation is in the form of (4.1). Hence, the methods employed in this section for the Legendre and Bessel functions may be applied to the remaining three cases considered by Stepanoff. In particular, expansions for Lamé and Mathieu functions can be found.

The case of second order equations in more than three variables offers no essential difficulty. For $n$ variables there would be $n-1$ generalized powers each with one index and one "power" with $n-1$ indices. This fact and the type of equation that can be considered are shown in Theorem (3.15).

\section{BiBLIOGRAPHY}

1. S. Bergman, $A$ formula for the stream function of certain flows, Proc. Nat. Acad. Sci. U.S.A. vol. 29 (1943) pp. 276-281.

2. - Pseudo harmonic vectors and their properties, Bull. Amer. Math. Soc. vol. 51 (1945) p. 657.

3. L. Bers and A. Gelbart, On a class of differential equations in mechanics of continua, Quarterly of Applied Mathematics vol. 1 (1943) pp. 168-188.

4. - On a class of functions defined by partial differential equations, Trans. Amer. Math. Soc. vol. 56 (1944) pp. 67-93.

5. W. Stepanoff, Sur l'equation de Laplace et certains systemes triples orthogonaux, Rec. Math. (Mat. Sbornik) N.S. vol. 11 (1942) pp. 204-238.

6. E. T. Whittaker and G. N. Watson, A course of modern analysis, New York, 1943.

7. R. von Mises and K. Friedrichs, Fluid dynamics, Brown University, 1942. Supplement.

BROWN UNIVERSITY, Providence, R. I. 